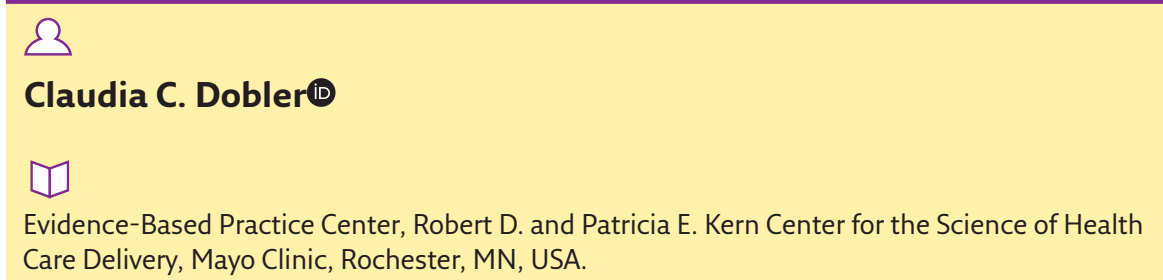

\title{
The IMPACT of triple versus dual single-inhaler therapy on exacerbations of COPD
}

\section{Journal club}

\section{Commentary on:}

Lipson DA, et al. Once-daily single-inhaler triple versus dual therapy in patients with COPD. NEngl J Med 2018; 378: 1671-1680.

\section{Context}

For a long time, the combination of an inhaled corticosteroid (ICS) and a long-acting $\beta$-agonist ( $L A B A)$ inhaler agent was considered the mainstay of treating chronic obstructive pulmonary disease (COPD) patients with severe airflow obstruction and frequent exacerbations. More recently, the role of ICS in the treatment paradigm of patients with severe COPD and frequent exacerbations has been challenged. This is mainly driven by: 1) the growing evidence of an increased risk of pneumonia associated with ICS [1]; 2) the introduction of combined dual long-acting bronchodilator inhalation therapy with a long-acting muscarinic antagonist (LAMA) and a LABA therapy as a plausible and likely superior treatment alternative [2]; and 3) the recognition that probably not all COPD exacerbation phenotypes stand to equally benefit from ICS therapy, e.g. patients with high blood eosinophil counts probably stand to benefit more from ICS therapy than those with low eosinophil counts [3]. The Informing the Pathway of COPD Treatment (IMPACT) study aimed to address the question of whether ICS in addition to dual therapy with LAMA/LABA can reduce moderate and severe acute exacerbations of COPD (AECOPD) compared with LAMA/LABA therapy alone [4].

\section{Methods}

This was a multicentre, randomised controlled trial that included patients aged $\geq 40$ years who either had a forced expiratory volume in $1 \mathrm{~s}\left(\mathrm{FEV}_{1}\right)$ that was $<50 \%$ of the predicted normal value and a history of at least one moderate or severe AECOPD in the previous year, or an $\mathrm{FEV}_{1}$ of $50-80 \%$ of the predicted normal value and at least two moderate or one severe AECOPD in the previous year. The study was conducted in 37 countries. Patients were randomly assigned to one of three treatment
Cite as: Dobler CC. The IMPACT of triple versus dual single-inhaler therapy on exacerbations of COPD. Breathe 2018; 14: 333-335. 
arms: 1) a once-daily triple combination therapy of fluticasone furoate (an ICS) $100 \mu \mathrm{g}$, umeclidinium (a LAMA) $62.5 \mu \mathrm{g}$ and vilanterol (a LABA) $25 \mu \mathrm{g}$; 2) a once-daily dual combination therapy of fluticasone furoate $100 \mu \mathrm{g}$ and vilanterol $25 \mu \mathrm{g}$; or 3) a oncedaily dual combination therapy of umeclidinium $62.5 \mu \mathrm{g}$ and vilanterol $25 \mu \mathrm{g}$. Each treatment was given in a single dry-powder inhaler (Ellipta, GlaxoSmithKline). The primary outcome was the annual rate of moderate or severe AECOPD during treatment. A mild AECOPD was defined as worsening of symptoms treated with increased salbutamol. A moderate AECOPD was one treated with antibiotics or systemic glucocorticoids, and a severe AECOPD was one resulting in hospitalisation or death.

\section{Main results}

Over a 3-year period, 10355 patients were randomised and included in the intention to treat analysis, of which $88 \%$ completed the trial. Patients on triple therapy with ICS/LAMA/LABA had a mean of 0.91 moderate or severe AECOPD per year during treatment, compared with 1.07 per year among patients on dual therapy with ICS/LABA and 1.21 per year among patients on dual therapy with LAMA/LABA. This resulted in a rate ratio of $0.85(95 \% \mathrm{Cl} 0.80-0.90)$ when comparing ICS/LAMA/LABA with ICS/LABA therapy and a rate ratio of $0.75(95 \% \mathrm{Cl} 0.70-0.81)$ when comparing ICS/LAMA/LABA with LAMA/LABA therapy. Therefore, the rate of moderate and severe AECOPD was significantly lower with ICS/LAMA/LABA therapy than with ICS/LABA or LAMA/LABA therapy. This effect was observed regardless of eosinophil level, although a greater reduction in the rate of moderate or severe AECOPD in patients on an ICS compared with those not receiving an ICS was observed in patients with eosinophil levels of $\geq 150$ cells $\mu \mathrm{L}^{-1}$. The annual rate of AECOPD was 0.95 (95\% Cl 0.90-1.01) with ICS/ LAMA/LABA therapy, $1.08(95 \% \mathrm{Cl} 1.02-1.14)$ with ICS/LABA therapy, and 1.39 (95\% Cl 1.29-1.51) with LAMA/LAMA therapy in patients with eosinophil levels of at least 150 cells $\mu \mathrm{L}^{-1}$.

All-cause mortality was significantly lower in the groups that received an ICS compared with the LAMA LABA group (50 patients, $1 \%$, in the triple therapy group, 49 patients, 1\%, in the ICS/LABA group and 39 patients, $2 \%$, in the LAMA/LABA group).

The rate of pneumonia was significantly higher in the groups that received an ICS compared with the LAMA/LABA group (9.6 and 9.7 versus 6.1 pneumonias per 100 patient-years in the ICS/ LAMA/LABA, ICS/LABA and LAMA/LABA groups, respectively).

\section{Commentary}

This study showed that triple inhalation therapy resulted in a significantly lower rate of moderate or severe COPD exacerbations than either dual therapy with ICS/LABA or LAMA/LABA. The certainty in the evidence provided by this study is lowered by features of the study design and the included study population. Many of the patients assigned to the LAMA/LABA group were stepping down in their treatment and had ICS abruptly withdrawn at the time of randomisation, which could have triggered an AECOPD. Also, patients with a history of asthma were included, which could explain the very high rates of AECOPD in the first month after randomisation in the LAMA/LABA group [5]. Thus, the benefit of triple therapy in comparison to LAMA/LABA therapy might have been overestimated in the IMPACT study. As outlined by the authors of the study, these study features probably also explain why the findings stand in contrast to those of the FLAME trial, which showed a benefit of LAMA/LABA over inhaled ICS/LABA therapy [2].

Of note, however, the TRIBUTE trial which was published in early 2018, and included 1532 patients in 17 countries, also showed that a single-inhaler triple combination of beclometasone dipropionate, glycopyrronium and formoterol fumarate (ICS/ LABA/LABA) significantly reduced the rate of moderate and severe AECOPD compared with a single-inhaler dual bronchodilator combination of glycopyrronium and indacaterol (LAMA/LABA) [6]. This effect was observed despite the fact that the TRIBUTE study excluded patients who were already on triple therapy, meaning that there was no step down in treatment, and also excluded patients with a current diagnosis of asthma.

In the IMPACT study, all-cause mortality was lower in the groups that received an ICS, but the study was not powered for this outcome, and the results should therefore be interpreted with caution, especially as they differ from the findings of the SUMMIT study [7]. The SUMMIT study was powered for the outcome of all-cause mortality and did not show a benefit in all-cause mortality when adding an ICS to a LABA (fluticasone furoate/vilanterol compared with vilanterol).

A recently published pooled analysis of mortality reported as safety outcome (as opposed to mortality reported as an efficacy outcome) in three landmark studies in patients with severe and very severe COPD found that the risk of nonrespiratory mortality was significantly reduced with extrafine ICS-containing treatments compared with ICS-free treatments (hazard ratio 0.65; 95\% $\mathrm{Cl} 0.43-0.97$ [ [8]. The authors argued that this potentially points towards a positive effect of ICS on comorbidities associated with COPD. The pooled analysis was, however, not based on a systematic review, and the limited focus on mortality reported as adverse outcome, while ignoring mortality reported as efficacy outcome, is problematic.

In the IMPACT study, the risk of pneumonia was significantly increased in patients on ICS treatment but was not associated with an increase in all-cause mortality in patients on ICS treatment, as outlined above. This finding is consistent with the result of a systematic review that found an increased relative risk of pneumonia of 1.61 (95\% Cl 1.35-1.93) 
associated with ICS treatment in COPD patients in 29 randomised controlled trials but no increase in all-cause mortality (relative risk $0.95 ; 95 \% \mathrm{Cl}$ 0.85-1.05) [1]. It has been speculated that ICS could have a dual effect: an adverse effect from the immunosuppressive properties of ICS and a mitigating effect from the anti-inflammatory properties of ICS [9]. While the immunosuppressive effects increase the risk of pneumonia, it is possible that the anti-inflammatory effects of ICS reduce the severity of an episode of pneumonia and therefore the pneumonia case fatality.

\section{Implications for practice}

Clinicians should only consider triple therapy in symptomatic patients with COPD who have frequent AECOPDs despite maximised bronchodilator therapy with a LAMA/LABA combination, as recommended by the $2018 \mathrm{Global}$ Initiative for Chronic Obstructive Lung Disease guidelines [10]. The potential benefit of reduced exacerbation frequency with ICS/LAMA/LABA treatment compared with LAMA/LABA treatment has to be weighed against the increased risk of pneumonia. COPD patients who are potentially eligible for ICS treatment based on the severity of their disease and frequent exacerbations, should be informed about potential benefits as well as harms of ICS treatment by the treating clinician. The patient and clinician should then determine together the best course of action for an individual patient taking into account the patient's informed preferences.

\section{Conflict of interest}

C.C. Dobler has nothing to disclose.

\section{Support statement}

C.C. Dobler is supported by a fellowship from the Australian National Health and Medical Research Council (NHMRC), APP1123733.

\section{References}

1. Festic E, Bansal V, Gupta E, et al. Association of inhaled corticosteroids with incident pneumonia and mortality in COPD patients; systematic review and meta-analysis. COPD 2016: 13: 312-326

2. Wedzicha JA, Banerji D, Chapman KR, et al. Indacaterolglycopyrronium versus salmeterol-fluticasone for COPD. N Engl J Med 2016; 374: 2222-2234.

3. Chapman KR, Hurst JR, Frent SM, et al. Long-term triple therapy de-escalation to indacaterol/glycopyrronium in patients with chronic obstructive pulmonary disease (SUNSET): a randomized, double-blind, triple-dummy clinical trial. Am J Respir Crit Care Med 2018; 198: 329-339.

4. Lipson DA, Barnhart F, Brealey N, et al. Once-daily singleinhaler triple versus dual therapy in patients with COPD. NEngl J Med 2018; 378: 1671-1680.

5. Suissa S, Drazen JM. Making sense of triple inhaled therapy for COPD. N EnglJ Med 2018; 378: 1723-1724.

6. Papi A, Vestbo J, Fabbri L, et al. Extrafine inhaled triple therapy versus dual bronchodilator therapy in chronic obstructive pulmonary disease (TRIBUTE): a double-blind, parallel group, randomised controlled trial. Lancet 2018; 391: 1076-1084.

7. Vestbo J, Anderson JA, Brook RD, et al. Fluticasone furoate and vilanterol and survival in chronic obstructive pulmonary disease with heightened cardiovascular risk (SUMMIT): a double-blind randomised controlled trial. Lancet 2016; 387: 1817-1826.

8. Vestbo J, Fabbri L, Papi A, et al. Inhaled corticosteroid containing combinations and mortality in COPD. Eur Respir J 2018; in press [https://doi.org/10.1183/13993003.01230-2018].

9. Festic E, Scanlon PD. Incident pneumonia and mortality in patients with chronic obstructive pulmonary disease. A double effect of inhaled corticosteroids? Am J Respir Crit Care Med 2015; 191: 141-148.

10. Global Initiative for Chronic Obstructive Lung Disease. Global strategy for the diagnosis, management, and prevention of chronic obstructive pulmonary disease. 2018 Report. https:// goldcopd.org/wp-content/uploads/2017/11/GOLD-2018v6.0-FINAL-revised-20-Nov_WMS.pdf Date last accessed: September 2, 2018 